\title{
Effets secondaires des androgènes
}

\author{
I. Mowszowicz
}

Service d'Endocrinologie et de Médecine de la Reproduction et Laboratoire de Biochimie B, Hopital Necker, PARIS

\section{RESUME}

Les androgènes utilisés à des doses 10 à 100 fois supérieures aux doses thérapeutiques provoquent des effets qui peuvent être classés en 4 catégories :1) Effets androgéniques : Chez l'homme, les androgènes provoquent atrophie testiculaire et azoospermie avec stérilité, réversibles en 2 à 3 mois à l'arrêt du traitement. Il serait utile de surveiller à long terme la prostate des sujets soumis à de fortes doses d'androgènes, une étude récente ayant montré une élévation des taux de PSA. Chez la femme, l'enfant et l'adolescent, les effets virilisants sont constants et irréversibles ce qui conduit à proscrire l'utilisation des stéroïdes androgènes/ anabolisants à forte dose chez ces sujets. 2) Effets toxiques : surtout liés à l'utilisation de stéroïdes $17 \alpha$-alkylés, ils atteignent essentiellement la fonction hépatique. 3) Effets sur le système cardiovasculaire : modifications des lipides et des lipoprotéines sanguines allant dans le sens d'une augmentation du risque athéromateux. 4) Effets comportementaux : les androgènes sont censés développer l'agressivité et l'endurance. Des manifestations de type psychotiques ont été rapportées ainsi que l'apparition d'une dépendance physique et psychologique. Toutefois chez l'homme, ces effets ne sont pas sérieusement documentés ; il semble exister un effet placebo important. En conclusion, chez l'homme adulte au moins, les effets androgéniques propres sont largement réversibles à l'arrêt des traitements et ne semblent pas comporter de risques graves. Par contre les effets potentiels à long terme : effets toxiques hépatiques, conséquences des modifications des lipoprotéines et effets éventuels sur le développement d'une pathologie prostatique androgénodépendante ne sont actuellement pas évalués.

Mots-clés : Stéroides anabolisants, stérö̈des androgènes, abus d'androgènes, effets secondaires, effets cardio-vasculaires, effets hépatiques, effets comportementaux.

Les androgènes sont utilisés en pratique médicale dans le traitement des hypogonadismes masculins pathologiques ou physiologiques (andropause). Ils permettent de rétablir des taux d'androgènes circulants normaux et ont pour but la reproduction des effets physiologiques de la testostérone. Ils sont, dans ces conditions, bien tolérés et sans effets secondaires majeurs. Par contre, l'utilisation détournée des mêmes molécules par les sportifs, dans le but d'améliorer leurs performances physiques (dopage), présente des risques non négligeables et d'autant plus difficiles à évaluer qu'il s'agit d'une pratique illégale et donc clandestine. De plus, les doses utilisées sont 10 à 100 fois supérieures aux doses thérapeutiques 
et viennent s'ajouter à une production d'androgènes normale chez ces sujets sains. Un troisième type d'utilisation est en cours d'évaluation et devrait permettre d'apprécier les effets de doses intermédiaires: il s'agit de la contraception masculine. Trois revues récentes tentent de faire le point sur les risques et les bénéfices de l'utilisation des steroïdes androgènes/anabolisants [3, 9, $16]$.

\section{MECANISME D'ACTION DES ANDROGENES}

Les androgènes comme toutes les hormones stéroïdes agissent par l'intermédiaire d'un récepteur, protéine soluble appartenant à la famille des récepteurs nucléaires [5]. Les stéroïdes pénètrent dans la cellule cible, probablement par diffusion passive et se lient au récepteur. Le complexe hormone récepteur se lie ensuite à des séquences spécifiques d'ADN, dans la zone promotrice des gènes régulés et induit la synthèse de protéines spécifiques [6]. Il faut rappeler que dans de nombreux tissus cibles, la testosterone (T), principal androgène circulant, est transformée par une $5 \alpha$-réductase en dihydrotestosterone ( DHT) dont l'affinité pour le récepteur est trois à cinq fois supérieure à celle de la testostérone, réalisant ainsi une amplification de l'action androgénique [11]; toutefois certains tissus, en particulier le muscle, n'ont qu'une faible activité $5 \alpha$-réductase et au contraire une forte activité 3-oxoréductase transformant la DHT en androstanediols ( $3 \alpha$ ou $3 \beta$ ) inactifs, de telle sorte que la concentration tissulaire en DHT est toujours très faible [13]. Dans ces tissus, c'est la testostérone elle même qui se lie au récepteur et exerce directement son action. Il n'existe qu'un seul récepteur des androgènes : la testostérone, la DHT, mais aussi les norstéroïdes et autres anabolisants se lient au (et agissent par l'intermédiaire du) même récepteur [10, 14]. Il n'est donc pas possible de dissocier complètement les actions des androgènes de celles des stéroïdes anabolisants (l'anabolisant pur n'existe pas) Enfin, certains stéroïdes androgéniques (en particulier les esters de la testostérone) sont métabolisés en un autre stéroïde actif, en particulier en estrogènes : ils agiront alors par l'intermédiaire du récepteur des estrogènes. Ce métabolisme peut se faire dans les tissus cibles ou en périphérie: étant donné les très fortes doses administrées, un faible taux de métabolisme produit une quantité non négligeable de métabolites [18].

\section{EFFETS SECONDAIRES DES ANDROGENES}

Ce sont les effets non désirés des androgènes: ils dépendent de la molécule utilisée et de la dose administrée. Les différentes conditions d'utilisation des androgènes sont rappelées sur le tableau 1.

- Dans l'hypogonadisme ou le traitement de l'andropause, il s'agit de compenser la diminution pathologique ou physiologique de la production d'androgènes pour ramener les taux d'androgènes circulants à des valeurs normales. L'effet recherché est essentiellement androgénique dans l'hypogonadisme, androgénique et anabolisant chez l'homme âgé.

- Dans le cas de la contraception masculine, il s'agit d'ajouter à une production normale, des doses supraphysiologiques de manière à bloquer l'axe hypothalamo-hypophyso-gonadique et donc inhiber la spermatogénèse.

- Les athlètes, enfin, utilisent des doses énormes, 10 à 100 fois supérieures aux doses utilisées en thérapeutique, et recherchent surtout les effets anabolisants; les effets androgéniques deviennent alors des effets secondaires.

Enfin, il faut signaler que si des études contrôlées des effets secondaires peuvent être, et ont été réalisées dans le cadre de l'utilisation thérapeutique des androgènes, 


\begin{tabular}{lccc}
\hline Indication & \multicolumn{2}{c}{ Effet Recherché } & Doses \\
\cline { 2 - 4 } & Andogénique & Anabolisant & \\
\hline HYPOGONADISME & ++ & + & SUBSTITUTIVES \\
$\begin{array}{l}\text { VIEILLISSEMENT } \\
\text { ANDROPAUSE }\end{array}$ & + & & SUBSTITUTIVES \\
$\begin{array}{l}\text { CONTRACEPTION } \\
\text { MASCULINE }\end{array}$ & + & & SUPRA \\
$\begin{array}{l}\text { DOPAGE } \\
\text { (illégal) }\end{array}$ & + & & PHYSIOLOGIQUES \\
\hline
\end{tabular}

ces études n'existent pas dans le cas de la prise, toujours illégale, de stéroïdes androgènes/anabolisants par les athlètes et l'interprétation des données existantes est encore compliquée du fait de l'utilisation cumulée de diverses molécules à des doses variables et progressivement croissantes [18].

\section{Molécules utilisées et mode d'admi- nistration}

Les molécules utilisées, toutes dérivées de la testostérone, appartiennent à 3 grandes catégories: les esters de la testostérone (estérification de l'hydoxyle en 17ß), les dérivés $17 \alpha$-alkylés, les molécules modifiées sur le noyau A [18] (Figure 1).

Le mode d'administration varie avec le type de molécule (Tableau 2). La testostérone ne peut pas être administrée par voie orale, car elle se métabolise trop rapidement, dès le premier passage hépatique. Même si l'esterification ralentit le métabolisme, et ce d'autant plus que la chaîne est plus longue, les esters de la testostérone seront administrés par voie parentérale. Une exception toutefois, l'undécanoate de testostérone, de par sa longue chaîne, est très liposoluble et est absorbé par la lymphe plutôt que dans la veine porte. Il peut donc être utilisé par

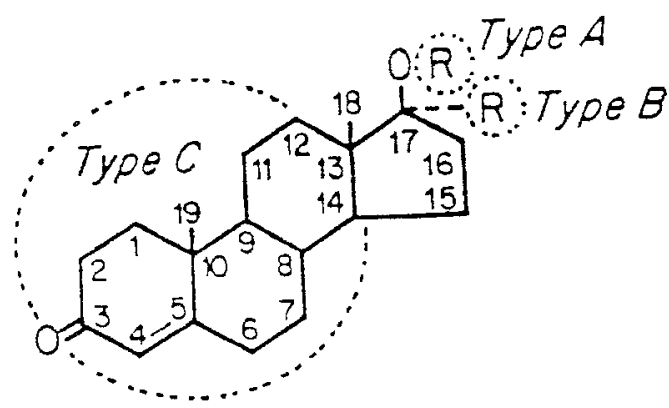

Type A : Androgènes estérifiés, Type B : Androgènes 17 $\alpha$-alkylés,

Type $\mathrm{C}$ : Androgènes avec modifications du noyau steroïdien.

Figure 1 : Différentes molécules androgèniques utilisées (voir tableau 2 pour des exemples de $R$ ).

voie orale. Il en est de même de la testostérone micronisée.

Les dérivés $17 \alpha$-alkylés peuvent être ou non modifiés sur le noyau A. Ils ne sont pas convertis en testostérone et lors de leur passage hépatique, ils donnent des métabolites particuliers qui permettent leur identification. Les norsteroïdes sont extrêmement puissants : leur affinité pour le récepteur est du même ordre que celle de la DHT du fait de la structure plane du noyau A [10]. 
Esters de testostérone

\begin{tabular}{|c|c|c|}
\hline $\mathrm{R}=\mathrm{COCH}_{2} \mathrm{CH}_{2}$ & propionate & parentérale \\
\hline $\mathrm{R}=\mathrm{COCH}_{2} \mathrm{CH}_{2}$ & cypionate & $\langle(»)+\cdots\rangle$ \\
\hline $\mathrm{R}=\mathrm{CO}\left(\mathrm{CH}_{2}\right)_{5} \mathrm{CH}_{3}$ & énanthate & «m!n») \\
\hline $\mathrm{R}=\mathrm{CO}\left(\mathrm{CH}_{2}\right)_{8} \mathrm{CH}=\mathrm{CH}_{2}$ & undécanoate & orale \\
\hline
\end{tabular}

Dérivés $17 \alpha-$ alkylés

sans modification du noyau stéroïdien

$\mathrm{R}=\mathrm{CH}_{3}$

Méthyltestostérone

orale

avec modification du noyau stéroïdien

- portant sur le noyau A

Fluoxymesterone

orale

Oxandrolone

Danazol

- Norstéroïdes

$\mathrm{R}=\mathrm{CH}_{2} \mathrm{CH}_{3}$

Noréthandrolone

orale

\section{Effets secondaires des androgènes}

Ils peuvent être classés en 4 catégories :

a) Effets androgéniques

Chez l'homme, administrés à doses supraphysiologiques, les androgènes provoquent toujours, du fait du retro-contrôle qu'ils exercent sur l'axe hypothalamo-hypophysaire, une chute des taux circulants de $\mathrm{LH}$ et de FSH aboutissant à une diminution de la secrétion de testostérone endogène, une diminution du volume testiculaire (20 à 30 $\%)$ et une azoospermie presque complète avec stérilité [18]. On peut également observer, à très fortes doses, une prise de poids et une augmentation de l'hémoglobine. Enfin, si les androgènes utilisés sont aromatisables (esters de la testostérone), les taux d'estradiol s'élèvent et peuvent s'accompagner d'une gynécomastie. Ces effets sont réversibles à l'arrêt du traitement mais l'hypogonadisme peut persister pendant 2 à 3 mois.

Il serait probablement utile de surveiller à long terme la prostate de ces sujets pour définir s'il existe un risque accru d'hypertrophie prostatique ou même de cancer de la prostate. Lors d'une étude récente, tentant d'évaluer les effets des androgènes à long terme et à doses supraphysiologiques, Tenover a administré à 13 hommes âgés de 57 à 76 ans, $100 \mathrm{mg} / \mathrm{semaine}$ d'enanthate de T, et ce, pendant 3 mois : le PSA s'élève de 2,1 à $2,7 \mathrm{ng} / \mathrm{ml}$ pendant la période de traitement, mais surtout, il n'est pas revenu à la normale 3 mois après l'arrêt du traitement [17]. D'autre part, Roberts et Essenhigh [15] ont décrit un cas d'adénocarcinome de la prostate chez un «bodybuilder» de 40 ans, qui avait pris de la methandrostenolone à long terme, sans toutefois qu'on puisse dire s'il s'agit d'un effet toxique de ce dérivé $17 \alpha$-alkylé, d'un effet androgénique ou du hasard.

Chez la femme, les effets virilisants des androgènes sont constamment observés : augmentation de la masse musculaire mais aussi acné, hirsutisme, perte de cheveux, hypertrophie clitoridienne, modifications de la voix. Chez l'enfant et l'adolescent, les mêmes effets virilisants peuvent être observés, avec en plus un risque de soudure prématurée des cartilages de conjuguaison. 
Ces effets sont irréversibles et de ce fait l'utilisation des stérö̈des androgènes /anabolisants à forte dose est à proscrire chez les femmes et les adolescents.

\section{b) Effets toxiques}

Ils sont surtout liés à l'utilisation de stéroïdes $17 \alpha$-alkylés et ils atteignent essentiellement la fonction hépatique (augmentation des phosphatases alcalines, des proteines plasmatiques, de la bilirubine conjuguée, diminution de la conjugaison des stéroïdes) pouvant se manifester parfois par un ictère. Ils peuvent également provoquer des tumeurs (peliose hépatique, hépatomes) [18].

\section{c) Effets sur le système cardiovasculaire}

Des modifications des lipides et des lipoprotéines sanguines sont constamment retrouvées avec les fortes doses. Le risque athéromateux semble augmenté comme en témoigne l'élévation des LDL et du cholestérol total et la baisse des HDL, proportionnelles à la dose d'androgènes administrée et parallèles à l'augmentation des androgènes plamatiques $[2,8]$. Le mécanisme de ces modifications n'est pas clair. Elles sont surtout associées à la prise de stéroïdes $17 \alpha$-alkylés et seraient peut-être dues à un effet toxique.

Un risque accru de thrombose par augmentation de l'agrégation plaquettaire a également été rapporté, soit par un mécanisme impliquant les prostaglandines : aumentation de la production de Thromboxane A2 (stimulant de l'agrégation plaquettaire) ou inhibition de la production de Prostacycline PGI2 (inhibiteur de l'aggrégation plaquettaire), soit par un mécanisme indirect lié à l'augmentation des LDL (augmentation de la sensibilité plaquettaire) ou du fibrinogène [7]. Enfin, 1 cas de mort subite par infarctus du myocarde a été rapporté [12].

\section{d) Effets comportementaux}

Les androgènes sont censés développer l'agressivité et l'endurance et c'est pour cette raison qu'ils sont utilisés par les sportifs. La réalité de ces effets «bénéfiques» n'est pas démontrée. Par contre, des manifestations de type psychotique (irritabilité, comportements violents et maniaques) ont été rapportées ainsi que l'apparition d'une dépendance physique et psychologique . Toutefois chez l'homme, ces effets ne sont pas sérieusement documentés. Deux études contrôlées récentes $[1,2]$ n'ont montré aucun effet de l'enanthate de $\mathrm{T}$ à la dose de $800 \mathrm{mg} /$ semaine, sur l'excitabilité, l'agressivité et l'activité sexuelle, pendant 8 semaines dans un cas, 16 à 20 semaines dans l'autre. Une des rares études en double aveugle [4], portant sur 27 jeunes finlandais âgés de 21 à 31 ans, étudiants et sportifs, répartis en 3 groupes comparables quant à la testostérone salivaire et recevant pendant une semaine soit $40 \mathrm{mg} / \mathrm{j}$ d'undécanoate de $T$, soit un placebo, soit rien, a montré un net effet du placebo sur les critères comportementaux étudiés (colère, irritabilité).

\section{CONCLUSION}

Chez l'homme adulte au moins, les effets androgéniques propres sont largement réversibles à l'arrêt des traitements et ne semblent pas comporter de risques graves. Par contre les effets potentiels à long terme sont actuellement mal évalués, que ce soit les effets toxiques hépatiques, les conséquences des modifications des lipoprotéines et les effets éventuels sur le développement d'une pathologie prostatique androgénodépendante. Il serait nécessaire, pour ces évaluations, de suivre à long terme des athlètes ayant «abusé» des androgènes pendant de longues périodes.

\section{REFERENCES}

1. ANDERSON R.A., BANCROFT J., WU F.C.W. : The effects of exogenous testosterone on sexuality and mood of normal men. J. Clin. Endocrinol. Metab. 1992, 75 : 1503-1507. 
2. BAGATELL C.J., HEIMAN J.R., MATSUMOTO A.M., RIVIER J.E., BREMNER W.J. : Metabolic and behavioural effects of high-dose exogenous testosterone in healthy men. J. Clin. Endocrinol. Metab. 1994, 79 : 561-567.

3. BARDIN C.W., SWERDLOFF R.S., SANTEN R.J. : Androgens : risks and benefits. J. Clin. Endocrinol. Metab. 1991, $73: 4-7$.

4. BJÖRKQVIST K., NYGREN T., BJÖRKLUND AC., BJÖRKQVIST S-E. : Testosterone intake and aggressiveness: real effect or anticipation? Aggressive Behavior, 1994, 20 : 17-26.

5. CHANG, C., KOKONTIS, J., LIAO, S. : Structural analysis of complementary DNA and amino acid sequences of human and rat androgen receptors. Proc. Natl. Acad. Sci. USA. 85, 1988, 7211-7215.

6. EVANS, R.M. : The steroid and thyroid hormone receptor superfamily. Science $240,1988,889-895$.

7. FERENCHICK G.S. : Anabolic-Androgenic steroids abuse and thrombosis : is there a connexion? Medical Hypothesis, 1991, $35: 27-31$.

8. GREGER N.G., INSULL W. Jr., PROBSTFIELD J.L., KEENAN B.S. : High-density lipoprotein response to 5a-dihydrotestosterone and testosterone in Macaca fascicularis : A hormone-responsive primate model for the study of atherosclerosis. Metabolism, 1990, 39 : 919-924.

9. HUHTANIEMI I. : Anabolic-Androgenic steroids - a double-edged sword? Int. J. Androl. 1994, 17 : 57-62.

10. KRIEG M., DENNIS M., VOIGT K.D. : Comparison between the binding of 19-nortestosterone, $5 \alpha-$ dihydrotestosterone, and testosterone in rat prostate and bulbocavernosus/levator ani muscle. J. Endocr. 1976, $70: 379-387$.

11. MAUVAIS-JARVIS P., MOWSZOWICZ I., KUTTENN F. : Significance of $5 \alpha$-reductase activity in human sexual differentiation. In : Sexual differentiation. Basic and cliniçal aspects. Serio M., Motta M., Zanisi M., Martini L. Eds. Serono Symp. Raven Press, New-York, 1984, 11, 247-260.

12. McNUTT R.A., FERENCHICK G.S., KIRLIN P.C., HAMLIN N.J. : Acute myocardial inarction in a 22-year-old world class weight lifterusing anabolic steroids. Am. J. Cardiol. 1988, $62: 164$.

13. MICHEL G., BAULIEU E-E. : Androgen receptor in skeletal muscle. J. Endocr. 1975, $65: 31$ P-32P.

14. MOWSZOWICZ I., RLAHI M., WRIGHT F., BOUCHARD Ph., KUTTENN F., MAUVAIS-JARVIS P.: Androgen receptor in human skin cytosol. J. Clin. Endocrinol. Metab. 1981, 52 : 338-344.

15. ROBERTS J.T., ESSENHIGH D.M. : Adenocarcinoma of prostate in 40-year-old body-builder. Lancet, $1986,2: 742$.
16. ROGOL A.D., YESALIS C.E. III : Anabolic-Androgenic steroids and athletes: what are the issues? J. Clin. Endocrinol. Metab. 1992, 74 : 465-469.

17. TENOVER J.S. : Effects of testosterone supplementation in the aging male. J. Clin. Endocrinol. Metab. 1992, 75 : 1092-1098.

18. WILSON J.D. : Androgen abuses by athletes. Endocr. Rev. 1988, $9:$ 181-198.

\section{ABSTRACT}

\section{Side effects of high-dose androgen administration}

\section{Mowszowicz}

Androgens, when used in medical replacement dosages, are well tolerated with no major side effects. In contrast, the illegal use by athletes of doses of androgens 10 to 100 fold higher than therapeutical doses can be responsible for a number of health hazards, all the more so since they are not medically controled. In addition, new usages of androgens at high doses are now emerging such as male contraception and hormone replacement therapy for andropause. Androgen side effects can be classified in 4 categories: il all cases they depend on the dose and the molecule used. 1) Androgenic side-effects are constant. In men, they include testicular atrophy and azoospermia leading to sterility. They are readily reversible in 2 to 3 months after cessation of treatment. However, potential long term effects on prostate should be evaluated. In women and adolescents, high doses of androgens will also produce androgenic side effects (increase of body mass, acne, deepening of the voice, hirsutism, balding) and in adolescents, premature epiphyseal fusion. These effects are often irreversible and should preclude high dose androgen administration in these subjects. 2) Toxic side-effects, especially due to the use of $17 \alpha$-alkyla- 
ted derivatives, bear mainly on liver function ; they involves pathological liver function tests and jaundice but can also include more serious hepatic complications such as peliosis hepatitis or hepatoma. 3) Effects on the cardiovascular system : blood lipids and lipoproteins change toward values promoting atherosclerosis are constant and an increased thrombogenic risk has also been reported. 4) Behavioural side-effects : Androgens are supposed to develop aggressivity and mental strength ; psychotic manifestations have been reported, but in the human these effects are poorly documented and there seemingly exists a strong placebo effect. In conclusion, in adult man at least, high doses of androgens seem to result in essentially reversible effects and androgen abuse do not involve major risks. However further studies are necessary to evaluate potential longterm effects such as liver toxic effects, significance of the cardiovascular changes and moreover, the potential development of an androgen-depen. dent prostate pathology.

Key-words : anabolic/androgenic steroid abuse, androgens, side-effects, cardiovascular effects, liver effects, behavioural effects. 